\title{
Políticas nacionales de salud y decisiones locales en México: el caso del Hospital Mixto de Cuetzalan, Puebla
}

\author{
María Beatriz Duarte-Gómez, MD, MSP, MSc, D ra en C, ${ }^{(1)}$ Viviane Brachet-Márquez, MS, PhD, ${ }^{(2)}$ \\ Roberto C ampos-N avarro, MD, MS PhD, ${ }^{(3)}$ G ustavo N igenda, MSC, PhD. ${ }^{(4)}$
}

\section{Duarte-Gómez MB, Brachet-Márquez V, Campos-Navarro R, Nigenda G. Políticas nacionales de salud y decisiones locales en México: el caso del Hospital Mixto de Cuetzalan, Puebla. Salud Publica Mex 2004;46:388-398. El texto completo en inglés de este artículo está disponible en: http://www.insp.mx/salud/index.html}

\section{Resumen}

Objetivo. Identificar la evolución del hospital del municipio de Cuetzalan a partir de la influencia que diversos elementos del contexto nacional e internacional han tenido so bre ella. Material y métodos. Estudio de caso, realizado en 2000 y 2001 sobre los Hospitales Mixtos en México -el de Cuetzalan, en el estado de Puebla, y el de Jesús María, en el estado de N ayarit-. Durante el trabajo de campo se obtuvo información a través de 72 entrevistas semiestructuradas con terapeutas alópatas e indígenas del hospital y con directivos de los diferentes niveles del sistema de salud. Además, se hizo una revisión documental, tanto de las políticas nacionales de salud para pueblos indígenas, como de las indigenistas, para relacionarlas con las cinco etapas organizacionales del hospital. Además de la autorización de los directivos estatales para el estudio, se pidió el consentimiento informado de los entrevistados. Resultados. El hospital fue creado en 1958 por la Secretaría de Salud con carácter bio médico, acorde con el indigenismo integracionista de la época, y continuó con ese carácter durante la administración inicial del Instituto $\mathrm{N}$ acional Indigenista. Pero las nuevas tendencias del indigenismo participativo y la sensibilidad y creatividad de algunos funcionarios del Instituto, sumadas a estrategias internacionales para lograr una ma-

\section{Duarte-Gómez MB, Brachet-Márquez V,}

Campos-Navarro R, Nigenda G.

National health policies and local decisions in Mexico:

the case of an intercultural hospital in Cuetzalan, Puebla.

Salud Publica Mex 2004;46:388-398.

The English version of this paper

is available at: http://www.insp.mx/salud/index.html

Este artículo forma parte de la tesis de la primera autora para optar por el grado de Doctora en Ciencias de la Salud Pública, en el Instituto N acional de Salud Pública de México, sobre el tema de la complementariedad entre medicina indígena y biomédica en los dos hospitales denominados "Mixtos" por la Secretaría de Salud en México, situados en Cuetzalan (Puebla) y Jesús María (N ayarit).

(1) Instituto Nacional de Salud Pública. Cuernavaca, Morelos, México.

(2) El Colegio de México. México, Distrito Federal, México.

(3) Facultad de Medicina. Universidad N acional Autónoma de México. México, Distrito Federal, México.

(4) Fundación Mexicana para la Salud. México, D istrito Federal, México.

Fecha de recibido: 19 de marzo de 2004 - Fecha de aprobado: 13 de julio de 2004

Solicitud de sobretiros: Dra. María Beatriz Duarte Gómez.Avenida D omingo Diez 1612 Casa 7, colonia Maravillas, 62250 Cuernavaca, Morelos, México.

Correo electrónico: maria_beatriz_duarte@ hotmail.com 
yor integración de las culturas indígenas, impulsaron su transformación, en 1990, en Hospital Mixto (que ofrecía las dos medicinas, la indígena y la alopática) con proyección regional. En la devolución del hospital a la Secretaría Estatal de Salud en 2000, que causó retrocesos temporales en el carácter mixto del hospital, primaron los criterios financieros sobre los socioculturales, mientras que en la actual versión del hospital como Hospital Integral con Medicina Tradicional influyeron, además de la iniciativa de funcionarios estatales, el reposicionamiento político de los indígenas en el país y la disponibilidad de recursos internacionales del Plan Puebla Panamá. Conclusiones. El análisis ratifica el carácter histórico del desarrollo de las organizaciones de salud, y las oportunidades que las coyunturas y políticas nacionales e internacio nales constituyen para impulsar modelos alternativos e interculturales de atención para la salud de los pueblos indígenas que respondan a sus necesidades. A pesar de que la medicina indígena y la biomédica han sido consideradas por algunos como irreconciliables, existen ejemplos - nacionales e internacionales- de experiencias en materia de articulación entre ellas, que permiten vislumbrar la posibilidad de crear nuevos pactos que avancen en la creación de servicios de salud verdaderamente interculturales, que sirvan de ejemplo para el resto del país y del continente. El texto completo en inglés de este artículo está disponible en:http://www.insp.mx/salud/index.html

Palabras clave: medicina intercultural; política de salud; México allopathic) with regional coverage. In 2000, the devolution of the hospital to the State Ministry of Health, based on financial rather than socio-cultural considerations, caused the temporary loss of its intercultural character. The last stage as an Integral Hospital with Traditional Medicine (from 2003 onwards) was due to a combination of state official initiatives and the new political stance acquired by the Mexican indigenous movement. The hospital is now part of a regional project of five such hospitals officially denominated Integral Hospitals with Traditional Medicine, to be financed by the Puebla - Panama Plan of regional development. Conclusions 0 ur results confirmed that health organizations follow a historical process in which selected national and international forces open opportunities to promote intercultural health models that respond to the needs of indigenous populations. Despite the formerly held belief that traditional and scientific medicines were incompatible, this study demonstrates the viability of intercultural health care models that may become a real possibility in the country, based on new conventions to establish alternative and intercultural health services, thereby setting an example for other regions and countries. The English version of this paper is available at: http://www.insp.mx/salud/index.html

Key words: intercultural medicine; health policy; Mexico os denominados hospitales mixtos en México representan una experiencia interesante, pero de difícil valoración en relación con la capacidad para ofrecer atención médica de buena calidad, desde el punto de vista técnico, y con sensibilidad cultural para ajustarse a las demandas específicas de la población indígena. El presente artículo, que hace parte de una investigación sobre los dos Hospitales Mixtos de Méxi$\mathrm{Co}^{*}$, tiene como objetivo principal analizar la evolución del hospital del municipio de Cuetzalan, del estado de Puebla, México, a partir de la influencia que diversos elementos del contexto nacional e internacional han tenido sobre ella.

El proceso del hospital de Cuetzalan ratifica el carácter histórico del desarrollo de las organizaciones de

\footnotetext{
* El estudio que se llevó a cabo como tesis para optar por el grado de Doctora en Ciencias de la Salud Pública en el Instituto Nacional de Salud Pública de México, se realizó entre 2000 y 2001 y tuvo como objetivos analizar el "Hospital Mixto" como estrategia de complementariedad en zonas indígenas de México -tomando como eje las relaciones entre los terapeutas tradicionales indígenas y los alópatas- y aportar elementos a los tomadores de decisiones sobre la utilidad y viabilidad de este modelo.
}

salud, y las oportunidades que las coyunturas y políticas nacionales e internacionales constituyen para impulsar modelos alternativos e interculturales de atención para la salud, entendiendo medicina intercultural como "la práctica y el proceso relacional que se establece entre el personal de salud y los enfermos, donde ambos pertenecen a culturas diferentes, $\mathrm{y}$ donde se requiere de un recíproco entendimiento para que los resultados del contacto (consulta, intervención, consejería) sean satisfactorios para las dos partes".*

A través del documento se establece una relación entre la evolución de las organizaciones de salud, los cambios de las políticas de salud en México, el contexto sociopolítico nacional e internacional, y las características personales de los tomadores de decisiones. Se concluye que la atención de la salud de los indígenas no ha sido una prioridad para el sector salud mexicano, quien la delegó durante un largo periodo al Instituto

* Campos- Navarro R. Una visión general sobre la medicina intercultural. En: Introducción a la medicina Intercultural. México, DF; 2003. Documento no publicado. 
Nacional Indigenista (INI). Sin embargo, la sensibilización social y el reconocimiento político nacional e internacional de los derechos de los pueblos indios, que han logrado los movimientos indígenas en México, en especial los de la última década, han influido positivamente en las recientes políticas gubernamentales de salud para indígenas, y permiten prever un avance en el proceso de garantizarles el acceso a servicios de salud que respeten sus diferencias e incluyan las prácticas tradicionales que se requieran para la recuperación de la salud y la prevención de la enfermedad.

Partiendo del reconocimiento de que las políticas públicas son formuladas y desarrolladas en un contexto social e histórico particular, y que incluyen en su proceso diferentes actores con diversos grados de participación, ${ }^{1,2}$ se revisan y analizan los principales cambios político-administrativos ocurridos en dicho hospital desde su creación en 1958 como Sanatorio Rural, a cargo de la entonces denominada Secretaría de Salubridad y Asistencia (SSA), hasta la transformación en Hospital Integral con Medicina Tradicional, de la Secretaría Estatal de Salud (SESA) de Puebla, en 2003.

Este documento describe de manera sucinta antecedentes de la historia de México relacionados de manera directa o indirecta con las políticas de salud para indígenas, antes de la creación del hospital; posteriormente, analiza cada una de las cinco etapas del hospital de Cuetzalan y su relación con el contexto, con énfasis en el cambio del INI a la Secretaría de Salud de Puebla, por ser en este momento de transición en el que se encontró el hospital al momento del estudio; finalmente, se plantea la discusión.

\section{Material y métodos}

La investigación de la que provienen los datos fue un estudio de caso múltiple.* Para la elaboración de este artículo se escogió el hospital de Cuetzalan para ejemplificar la influencia de los cambios en las políticas de salud y en el contexto social sobre un hospital municipal, por ser más antiguo que el de Jesús María y haber tenido múltiples cambios en su historia organizacional. Los datos de la evolución del hospital provienen casi en su totalidad del trabajo de campo de la investigación realizado entre 2000 y 2001, principalmente de las 35 entrevistas semiestructuradas individuales con terapeutas tradicionales y biomédicos del hospital, y con direc-

\footnotetext{
* El estudio de caso múltiple, en este caso el estudio de los dos hospitales denominados Mixtos en México, está descrito por varios autores como una forma de hacer el estudio más robusto y proveer una evidencia más convincente de los resultados. ${ }^{3,4}$
}

tivos del sector salud y del INI, tanto del ámbito nacional como del estatal y municipal, así como de dos reuniones de validación de resultados, y de la observación y la revisión de documentos. Alguna información adicional sobre los cambios más recientes del hospital y sobre los primeros antecedentes se obtuvieron en 2003, a partir de nuevas entrevistas con directivos de la Secretaría de Salud del ámbito federal (SSA), de la SESA de Puebla y con funcionarios y ex funcionarios del INI, además de la revisión documental sobre la historia de las políticas para indígenas en México. Los resultados de la investigación se reportan en dos artículos más, uno sobre el concepto de complementariedad aplicada a las políticas de salud intercultural y otro que resume los resultados del estudio en términos de la relación entre los dos tipos de terapeutas. ${ }^{*, 5}$

\section{Antecedentes}

\section{Políticas de salud para indígenas en México, un breve recuento histórico}

Con la llegada de los españoles al territorio de lo que hoy es México se inició un proceso de dependencia y sometimiento de los grupos nativos a la cultura occidental, y a la vez de intercambio epidemiológico y cultural.

En los siglos siguientes, la hoy denominada medicina biomédica -también nombrada galénica, occidental, científica, moderna- se desarrolló al servicio de la casta dominante, mientras que la indígena fue subordinada, desplazada y, en ocasiones, perseguida. ${ }^{6}$ La escasa atención biomédica a los indios estuvo a cargo de órdenes religiosas que crearon hospitales, algunos de ellos exclusivos para indígenas, en donde se practicaba la medicina intercultural. $\ddagger, 7$

Diversos grupos indígenas se concentraron en las denominadas "zonas de refugio"8 en las cuales más tarde se focalizarían las actividades de salud institucionales. Los gobiernos republicanos no particularizaron la salud de los pueblos indígenas y clausuraron hospitales como el Real Hospital de San José de los Naturales, considerado como el mejor hospital para indios de la Nueva España. $\ddagger$

Los ideales de la época de la Reforma, del hombre libre y creativo que elevaría el país a la altura de Euro-

\footnotetext{
* Duarte-Gómez MB. Is complementarity between indigenous and western medicine possible?- The case of the intercultural hospitals in Mexico; 2002. Documento no publicado.

‡ Campos- Navarro R. Una visión general sobre la medicina intercultural. En: Introducción a la medicina Intercultural. México, DF; 2003. Documento no publicado.
} 
pa, no produjeron mayores cambios en la situación de vida del indígena ${ }^{9}$ quien siguió colonizado y explotado, esta vez por criollos y mestizos. ${ }^{10-12}$ Con base en la idea de que la cultura indígena frenaba el "progreso", se intentaba integrar una nación homogénea, excluyente de la pluriculturalidad., ${ }^{9}, 13$ Durante el porfiriato no hubo una política de salud como tal, sino el deseo de "corregir el atraso científico y alcanzar a las grandes naciones" a través del desarrollo de algunos hospitales urbanos. ${ }^{14}$ Sin embargo, las epidemias de paludismo y fiebre amarilla que estaban entorpeciendo el comercio, obligaron, a partir de 1902, a realizar campañas verticales para su control y a crear agencias especializadas en higiene en puertos y fronteras.

A partir del inicio de la Revolución de 1910 se produce una serie de eventos, acuerdos y políticas que influyen sobre el proceso de institucionalización de los servicios de salud, entre los cuales destacamos:

- El inicio del denominado indigenismo, inicialmente planteado por Gamio, ${ }^{15}$ en 1916, con su libro Forjando Patria en el cual sintetiza todas las corrientes del indigenismo porfiriano. Se sostiene que la cultura indígena es la causa de su propio rezago, y se propone la integración de razas y culturas a través de la educación y del intercambio que dará lugar a una cultura nacional homogénea, aunque existieron diversas tendencias sobre el abordaje de esta pluriculturalidad y la forma de concebir y lograr la cultura nacional. ${ }^{8-10}$ Algunos valores y raíces indígenas se consideraron símbolos de la nacionalidad mexicana, ${ }^{9,16}$ pero fueron reducidos a un mero folclor, ${ }^{11}$ salvo el estudio de las plantas medicinales del que se han ocupado desde la Conquista grupos o personas interesadas en el tema de la herbolaria terapéutica utilizada por la medicina indígena.

- Hacia los años 20 del siglo XX, el Departamento de Salud Pública se acercó a la población general a través de las campañas para erradicar endemias y epidemias y mejorar el ambiente físico y cultural. La medicina "galénica o científica" curativa se ejercía de forma principalmente privada, lo cual la hacía inaccesible para el indígena, desde el punto de vista económico, cultural y geográfico. ${ }^{6,17}$ Por su parte, los indígenas conservaban sus tradiciones respecto al proceso salud/enfermedad/atención, y reinterpretaban, asimilaban o rechazaban los elementos de la nueva cultura médica, dando lugar a un modelo de transición descrito por Redfield, en el cual pacientes y curanderos acudían a una $u$ otra medicina. ${ }^{18}$

- Resultado de las reflexiones del Primer Congreso Nacional de Higiene Rural, llevado a cabo en 1936, se crearon el servicio social para pasantes de medicina y la Escuela Superior de Medicina Rural, ya extinta, adscrita al Instituto Politécnico Nacional, como una estrategia para garantizar la disponibilidad de médicos capacitados para trabajar en comunidades rurales y entender la cultura indígena. ${ }^{6}$

- La política indigenista de esa época se instrumentó a través de organismos especializados en la acción indigenista, como el Instituto Nacional Indigenista, creado en 1948, para asumir la conducción del llamado "desarrollo integral" en regiones indígenas, a través de los Centros Coordinadores. Estos organizaron unidades de salud atendidas por médicos y por promotores indígenas de salud que tenían como objetivo introducir la medicina biomédica* y "modificar actitudes y creencias que impidieran la aceptación de los beneficios de la medicina científica" ${ }^{8}$ Paralelamente, se intenta sistematizar los servicios sanitarios preventivo-asistenciales de manera permanente en todo el país. ${ }^{19}$ Sin embargo, sobre la situación de salud de los indígenas en esa época poco puede decirse, porque se carece de estadísticas adecuadas. ${ }^{6,17}$ La idea de medicina intercultural y básicamente preventiva de Aguirre-Beltrán, uno de los pioneros de la antropología médica en México, tenía hasta esta época una orientación culturalista y una finalidad de asimilación: la de abrir el camino para llevar la medicina pública o socializada sin obstáculos culturales. Esto requería estrategias para integrar esta medicina al contexto intercultural de las personas, incluir albergues junto a los hospitales, que permitieran el acompañamiento familiar, la intervención del terapeuta tradicional, el trabajo en equipo multidisciplinario, la educación higiénica con lenguaje adecuado, la visita domiciliaria y la utilización de los resultados de la investigación antropológica para ilustrar al personal de salud. ${ }^{6,8}$

Es en este contexto en el cual surge en 1958 la primera unidad de salud de Cuetzalan, cuya evolución en los siguientes 45 años describiremos a continuación.

\section{Resultados}

\section{La evolución del hospital de Cuetzalan}

El hospital está situado en el municipio de Cuetzalan que hace parte de la Sierra Norte del estado de Puebla;

\footnotetext{
* Campos- Navarro R. Una visión general sobre la medicina intercultural. En: Introducción a la medicina Intercultural. México, DF; 2003. Documento no publicado.
} 
el grupo étnico mayoritario es el nahua y, en menor número, el totonaca. La cabecera municipal es sitio de afluencia e intercambio comercial para los indígenas de ese y otros municipios aledaños.

Se pueden distinguir en su evolución cinco etapas en función de la pertenencia institucional y de su organización intercultural: la primera, que va desde su creación como Hospital Rural, en 1958, hasta 1978. La segunda, va de este último año cuando fue entregado al INI, quien lo organizó como Hospital de Campo, hasta 1990. La tercera, a partir de 1990, en la cual le fue conferido el carácter de Hospital Mixto por la inclusión de un área de medicina tradicional. La cuarta, va de 2000, año cuando fue transferido a la Secretaría Estatal de Salud y en el que se hizo el estudio, hasta 2003 cuando comienza la quinta etapa con la nueva denominación de Hospital Integral con Medicina Tradicional.

De cada una de las etapas se hará una breve descripción, seguida de algunos elementos del contexto nacional e internacional que se consideraron importantes.

\section{La primera unidad de salud de la Secretaría de Salubridad y A sistencia (1958-1978)}

Según datos aportados por la Secretaría de Salud de Puebla, en 1958 la Secretaría de Salubridad y Asistencia creó en Cuetzalan un Hospital Rural que prestaba servicios médicos de primer nivel de atención y algunos de segundo. Su construcción coincide con una de las etapas de mayor crecimiento de la infraestructura de la SSA en el área rural, la cual, según Hernández, no solamente tuvo como objetivo proyectar la imagen de un estado de bienestar, sino tratar de disminuir el flujo migratorio del campo a la ciudad. ${ }^{20}$

Paralelamente, en el ámbito internacional se avanzaba en el derecho a la autonomía, con acuerdos como la Resolución 1514 de la Organización de Naciones Unidas (ONU), en 1960, que estableció la libre determinación de los pueblos. Surgen políticas y acuerdos internacionales que se refieren de manera directa o indirecta a la vida y la salud de los indígenas.

A partir de 1968, se abría espacio el indigenismo participativo en contraposición al indigenismo de incorporación y a la imposición de valores biomédicos, con representantes como Warman, Bonfil y Stavengahen. Además, se empezaron a consolidar los movimientos indígenas en su lucha por la autonomía.

La Secretaría de Salud como responsable de la salud de los mexicanos sin seguridad social, incluyendo a los indígenas, aumenta la cobertura de servicios de salud curativa de tipo biomédico de manera paulatina en las comunidades indígenas principalmente a tra- vés del personal médico del INI, y posteriormente de la misma Secretaría de Salud y del Instituto Mexicano del Seguro Social -IMSS- a través del programa IMSSCoplamar* (hoy IMSS-Oportunidades), creado en 1979, sobre el cual Aguirre Beltrán opinaba que en este programa, lo económico había desplazado a los factores antropológicos y culturales indispensables para entender el proceso salud/enfermedad/atención. ${ }^{6}$

\section{EI Hospital de Campo del INI (1978-1989)}

Como parte del proceso de institucionalización de la política indigenista a cargo del INI, el hospital fue entregado por la SSA a éste en 1978, quien lo reinauguró como "Hospital de Campo" del Centro Coordinador de Zacapoaxtla. Esta unidad tenía un enfoque biomédico y asistencialista como correspondía al indigenismo asimilacionista de la época, lo cual se hace evidente en el testimonio de uno de los médicos directores del hospital en ese periodo:

[...] al llegar cambié el manual de normas técnicas por uno más social. Aceptaba que entraran el curandero o la partera si la familia lo solicitaba. Igualmente, permitía que entraran los familiares y aprovechaba para darles pláticas de salud. Apoyé el trabajo de la cooperativa indígena en su programa de salud regional, capacitando promotores de salud y haciendo consulta en las casas de la cultura, que se convirtieron también en casas de la "curación". Había apertura del INI federal para este tipo de propuestas. Sin embargo, fui desautorizado y trasladado por el INI delegacional [...] (entrevista con ex director del hospital).

Esta decisión del nivel regional nos muestra la dificultad para la operación de las nuevas tendencias nacionales del INI y de los acuerdos internacionales, que si bien no garantizan el cambio en las relaciones de los indígenas con la sociedad dominante, constituyen una herramienta para exigir la garantía de sus derechos en materia de salud; es el caso de la Declaración de Alma Ata $^{21}$ (1978), adoptada como modelo nacional, que promueve la atención primaria como estrategia para la ampliación de la cobertura de servicios de salud, utilizando los recursos de la comunidad, entre ellos los de la medicina tradicional, y el Convenio 169 de la Organización Internacional del Trabajo-OIT- sobre Pueblos Indígenas y Tribales (1989), ${ }^{22}$ que establece compromisos de los

\footnotetext{
* Coordinación General del Plan Nacional de Zonas Deprimidas y Grupos Marginados.
} 
gobiernos en temas como cultura, autonomía, identidad, tierras, educación, trabajo, salud y seguridad social.

\section{La transformación en Hospital Mixto (1990-2000)}

En 1990, Arturo Warman en la Dirección del INI, y Carlos Zolla en la Subdirección de Salud del mismo Instituto, ambos representantes de la corriente del indigenismo participativo, decidieron aprovechar la aceptación que tenía el Hospital entre las comunidades indígenas de Cuetzalan para hacer un programa regional de salud.* Así, el hospital no fue entregado nuevamente a la SSA como algunos sugerían, dado el proceso de descentralización de los servicios de salud que venía siendo promovido desde esta Secretaría y al cual no se sumaría el estado de Puebla sino varios años después.

Esta apertura hacia las comunidades de la región permitió ampliar la cobertura de los servicios básicos de salud a las localidades, con promotores de salud de la misma comunidad, teniendo en cuenta las estrategias de Atención Primaria en Salud y de los Sistemas Locales de Salud -SILOS- promovidas desde la Organización Panamericana de la Salud/Organización Mundial de la Salud (OPS/OMS). Se propuso la prestación regular de servicios de medicina tradicional dentro del hospital como una forma de acercarse culturalmente a la población, y de facilitar el uso complementario de las dos medicinas:

Dado el cariño y el sentido de pertenencia de la comunidad al hospital, propuse la creación de un hospital indígena que incluyera la medicina tradicional y la creación de un SILOS [Sistema Local de Salud] (directivo federal del INI).

Se hicieron las adaptaciones físicas que los terapeutas tradicionales consideraban necesarias:

[...] les preguntamos a los curanderos, ¿qué no les gusta de este hospital?, ahí uno se animó y dijo: que en este hospital no se reza... entonces necesitan que en cada consultorio haya un lugar para rezar. ¿Y las parteras qué dicen? Que cada que venimos a atender una paciente el doctor nos toma como su chacha, ahí él manda, nosotros somos como sus asistentes y no compartimos la forma en que los doctores atienden los partos. Conclusión: se necesitaban dos salas de parto [...] fue cuando metimos el temazcal y el baño de vapor y con las

\footnotetext{
* Información obtenida de la entrevista con funcionario del INI del nivel federal.
}

parteras empezamos a diseñar su sala de expulsión (directivo INI federal).

Otras adecuaciones fueron la creación de un jardín botánico y la utilización de formas de registro para que los(as) terapeutas tradicionales informaran de sus actividades en el hospital y pudiera darse lugar a una "epidemiología sociocultural" que incluyera el análisis de ambos tipos de diagnóstico. Se construyó un anexo para la medicina tradicional, con un consultorio para las parteras y otro para curanderos y hueseros, con su altar, una sala con camas, oficina, baño, temazcal, laboratorio para preparar medicamentos, sala de reuniones y bodegas. La pertenencia del hospital al INI facilitó el trabajo con el personal de salud biomédico para romper las barreras y prejuicios frente a la medicina tradicional, propias de su formación, y con los(as) médicos(as) tradicionales para romper el escepticismo

El INI propició la creación de una organización de médicos tradicionales -curanderas(os), parteras y hueseros(as)- en la zona a través de su programa de apoyo a la medicina tradicional, dando como resultado la formación de una organización de cerca de 120 terapeutas tradicionales del municipio y la creación de su centro de medicina tradicional-Masehualpajti- en 1991, con sede en el hospital; además colaboró con recursos económicos para facilitar las reuniones y el desplazamiento de los médicos tradicionales a dar consulta, y participó en la capacitación y dotación para la preparación de sus medicamentos.

La unidad de salud se convirtió en Hospital Mixto; los médicos tradicionales de la región empezaron a dar consulta en sus instalaciones de manera organizada y regular, al mismo tiempo que se ampliaba la oferta de recursos de salud biomédicos con diversos apoyos de organizaciones no gubernamentales, Institutos Nacionales como los de Nutrición y Pediatría y varias universidades. El Presidente Salinas de Gortari, en una visita al hospital, propuso crear un mayor número de éstos en el país, aunque solamente se construyó uno más, en Jesús María (Nayarit).

Según los informantes del INI, a partir de 1994 el presupuesto para el hospital fue haciéndose cada vez más insuficiente para garantizar el suministro adecuado y oportuno de medicamentos, la actualización de los equipos, el recurso humano necesario y el apoyo económico para los terapeutas tradicionales. Esto redujo la consulta de medicina tradicional que se había ofrecido diariamente, a los días de mercado (jueves y domingo).

Tras casi 10 años de funcionamiento como Hospital Mixto del INI, la Coordinación Nacional de Atención a Población Vulnerable, de la SSA, gestionó el traslado a la Secretaría Estatal de Salud, aprovechando 
la política de descentralización y la coyuntura del Programa de Ampliación de Cobertura de servicios básicos (PAC), para población no asegurada, financiado con un crédito del Banco Mundial.

A escala internacional esta década fue productiva en declaraciones y acuerdos sobre los pueblos indígenas como el Taller Hemisférico sobre Salud y Pueblos Indígenas, realizado en Winnipeg (Canadá) en 1993, y su declaración correspondiente; los acuerdos de la Cumbre de la Tierra en 1992; la resolución de la Cumbre de las Américas en 1994; la Iniciativa de Salud de los Pueblos Indígenas de la OPS/OMS, en 1992, y la declaración del Decenio Internacional de los Pueblos Indígenas del Mundo, a partir de 1994.

En estos y otros acuerdos y políticas sobre la salud de los pueblos indígenas, los países miembros de la Organización de Estados Americanos (OEA), de la ONU y de la OPS/OMS, se comprometieron a superar las barreras para el acceso a los servicios, a operativizar el concepto de salud integral e integrada, a reconocer el saber de las culturas nacionales y a generar mecanismos de interrelación respetuosa y complementaria, todo lo cual ha sido insuficientemente desarrollado en México.

\section{La entrega a la Secretaría Estatal de Salud y su nueva denominación de Hospital Integral (2000-2002)}

A partir de enero de 2000, la Secretaría Estatal de Salud se hizo cargo del hospital y lo organizó presupuestal y funcionalmente como un Hospital Integral más, sin tener en cuenta sus peculiaridades de hospital mixto, su historia, y el contexto cultural de la región, demostrando la falta de coordinación intersectorial en el manejo de las políticas para el trabajo en comunidades indígenas. Al respecto comenta uno de los directivos estatales de salud:

Cuando la Secretaría de Salud recibe Cuetzalan, no sabía lo que recibía. El presupuesto siguió dándose como un hospital alópata. Se recibió como tal, y lo que había trabajado el INI se vino hacia abajo. No recibimos un Hospital Mixto sino uno alópata [...] Y en los presupuestos nunca se reflejó nada de esto (funcionario SESA. Puebla).

Aunque durante su vinculación al INI el hospital sólo había cumplido con algunas de las características de interculturalidad que se han propuesto, como la posibilidad de acceso a la medicina tradicional, ${ }^{6,23}$ había logrado mantener el sentido de pertenencia por parte de la comunidad y de los terapeutas tradicionales, ra- zón por la cual los cambios no consensuados crearon inconformidad, tanto en unos como en otros.

[...] como ahora ya cambiaron, como ya no es INI, es sanidad y casi no quieren trabajar junto con nosotros (huesero, P18 Cuetzalan).

[...] cuando estuvo el doctor Raúl, el doctor Hilario, el doctor Lara, todos estuvo bien porque todos trabajábamos bien, todos éramos iguales, no había diferencias; claro, sí había diferencias porque ellos tienen otro tipo de medicina, pero había más respeto, había confianza [...] (curandera, P6 Cuetzalan).

El traslado fue traumático en términos de interculturalidad, pero mejoraron las condiciones técnicas de prestación de servicios de salud biomédicos por la mayor asignación presupuestal que permitió ampliar la planta de personal y mejorar el equipamiento.

La sensibilización y capacitación para el trabajo intercultural se había dado al personal de salud que trabajaba para el hospital del INI, pero no había llegado a los directivos de las Secretarías de Salud estatal y federal, lo cual explica la carencia de orientación a las nuevas directivas del hospital para dar prioridad al manejo intercultural (excepto en la continuidad del programa de parteras) y a la relación con el área de medicina tradicional. Esta continuó funcionando, pero de manera marginal, con serias deficiencias de comunicación entre las directivas del hospital y las de la organización de terapeutas tradicionales.

El 15 de junio de 2001, el Plan Puebla Panamá (PPP) fue presentado por el Presidente de México y aprobado por los de Centroamérica. Este plan se presenta con ocho objetivos básicos, uno de los cuales es el desarrollo humano y social en el que se incluye un proyecto regional de salud. ${ }^{24,25}$ El gobierno de Puebla, como parte de un programa estatal de ecoturismo relacionado con este Plan, tomó la iniciativa de construir un nuevo Centro de Medicina Tradicional en Cuetzalan, en un terreno propiedad de la organización de terapeutas tradicionales (Masehualpajti), cerca del hospital. A la fecha, el Centro no ha sido terminado por falta de presupuesto y no es claro cómo su funcionamiento afectará la presencia de estos terapeutas en el hospital.

\section{La denominación de Hospital Integral con Medicina Tradicional (2003-hasta la fecha)}

Al mismo tiempo que el hospital decaía como Hospital Mixto, y el gobierno de Puebla gestionaba la construcción del nuevo Centro de Medicina Tradicional, el equipo de Planeación de la SESA aprovechó el objetivo 
relacionado con salud del PPP para presentar en el mismo año el proyecto de "Hospitales Integrales con Medicina Tradicional". Entre sus objetivos estaba el de mejorar la baja utilización de los servicios de los hospitales de cinco zonas indígenas de Puebla, con influencia social y geográfica en otros estados colindantes como Guerrero y Veracruz. El proyecto fue aprobado sin obstáculos a escala estatal y, luego, en el Congreso Federal. $\mathrm{Al}$ respecto los funcionarios estatales comentan:

[...] yo creo que el movimiento indígena sensibilizó a la sociedad en general y a uno mismo, sobre la situación de marginación de los indígenas en el país y despertó el deseo de hacer algo por mejorarles la situación. Seguramente también a los políticos que aprobaron el proyecto en el Congreso sin obstáculos, lo cual probablemente no se hubiera dado unos años atrás. Además, nos sentimos respaldados por las políticas de la OPS, las de la Presidencia de la República y las de la Dirección de Medicina Tradicional de la Secretaría de Salud (directivo SESA, Puebla).

El proyecto contempla, para cada hospital, aumento de personal biomédico y su sensibilización y capacitación para el trabajo intercultural. También prevé el mejoramiento del equipo y de la infraestructura para la medicina biomédica, y la introducción de los terapeutas tradicionales en la oferta de servicios. Los médicos tradicionales de los cinco municipios fueron consultados para las obras de remodelación y adecuación de estos hospitales, entre ellos los del área de Cuetzalan. En este último caso, en el que ya se tenía el componente de la medicina tradicional, se reestructuró su funcionamiento, incluyendo apoyo económico para terapeutas tradicionales no pertenecientes a la organización que ofertaba los servicios desde su inicio como Hospital Mixto, y la presencia de asesores bilingües que sensibilizan al personal de salud de los hospitales para el trabajo intercultural, lo cual refuerza la capacitación contratada con un grupo de expertos. Esta estrategia concuerda con la planteada por la Secretaría de Salud federal:

Vamos a proponer una Norma Oficial Mexicana para que todo el personal de salud que vaya a trabajar en zonas indígenas tenga que certificarse como habilitado para eso [...] Creemos que va a ser bien recibida porque hay una propuesta similar del grupo interinstitucional (directivo 1, SSA).

El estado de Puebla tiene también, dentro de su proyecto, la idea de avanzar en el tema de legalización del ejercicio de la medicina tradicional, en lo cual hay concordancia con la Secretaría de Salud que propone como ejemplo y referencia la Ley Indígena de Oaxaca:

A todos los estados que quieren incursionar en la legalización, les damos los documentos del Diario Oficial de Oaxaca y los enviamos con los que hicieron allá el cabildeo (directivo 1, SSA).

A pesar de las propuestas del indigenismo respecto al reconocimiento de la existencia y utilización de la medicina tradicional en las comunidades indígenas, y de las recomendaciones internacionales de la OPS/OMS que se venían generando desde antes del año 2000, fue hasta 2001, tras los movimientos indígenas de la última década, cuando confluyeron las condiciones para este proyecto estatal de medicina tradicional en los hospitales de zonas indígenas del estado de Puebla. Además de la sensibilidad de funcionarios de salud estatales, otra condición favorable fue el compromiso político del nuevo gobierno federal para atender las zonas indígenas a través de la creación de una instancia gubernamental que lo asesore, y que represente los intereses de los indígenas en su gabinete, y por consiguiente, en los programas de cada sector gubernamental. La opinión de un directivo de la SSA sobre las razones sociopolíticas de este compromiso son las siguientes:

La primera y más importante es que el levantamiento indígena de 1994 vino a poner en primer plano político del país la situación y la condición de los indígenas... yo siento que esta administración cuando llega al poder en 2000 y el Presidente Fox desde su campaña hablaba de la deuda histórica con los pueblos indígenas, lo tuvo que hacer realidad a la hora de tomar el poder [...] (directivo 2 , SSA).

Las orientaciones de la Presidencia de la República dan lugar a la creación, en 2001, de la Coordinación de Salud y Nutrición para Pueblos Indígenas, en la Secretaría de Salud, y de una Dirección de Medicina Tradicional que, además de las razones políticas mencionadas, se justifican por

El rezago en salud de la población indígena frente al resto de la población y porque la medicina tradicional existe y la gente la utiliza, de manera que debemos tener una mejor comunicación con los terapeutas tradicionales y reconocerlos como prestadores de servicios $[\ldots]$ (directivo 2, SSA).

Sin embargo, la iniciativa de aumentar el número de Hospitales con Medicina Tradicional, en Puebla, pro- 
vino del mismo estado, sin intervención directa de la SSA federal:

Fue una idea del estado [de Puebla] De hecho, no compartimos la idea de los Hospitales Mixtos. En la Dirección de Medicina Tradicional tenemos una posición clara en ese sentido. No creas que vamos a cerrar los que ya hay. En esta Confederación los estados, en salud y en otras áreas, son libres y soberanos... Proponemos evaluar los que se crearon y saber si lograron lo que se proponían (directivo 1, SSA).

En resumen, el hospital fue creado con carácter biomédico, acorde con el indigenismo integracionista de la época, mientras que en el cambio, en 1990, a un Hospital Mixto con proyección regional, en donde se practicarían, tanto la medicina biomédica como la tradicional indígena, se conjugaron las nuevas tendencias del indigenismo participativo, la sensibilidad, experiencia y creatividad de algunos funcionarios del INI y algunas estrategias de la OPS/OMS. En la devolución del hospital a la Secretaría Estatal de Salud los criterios financieros primaron sobre los socioculturales, mientras que en la actual versión del hospital influyeron, además de la iniciativa de funcionarios de la SESA, el reposicionamiento social de los indígenas en el país y la disponibilidad de recursos internacionales.

\section{Discusión}

Dentro de las políticas de salud, el hospital de Cuetzalan representa un intento de llevar la medicina a las regiones indígenas y poner en práctica la teoría indigenista y las recomendaciones sobre la interculturalidad y la integralidad en la atención. Sin embargo, el proyecto original cambió de orientación en cinco momentos, según el contexto histórico, principalmente en función de influencias sociales, políticas -nacionales e internacionales-, y personales.

En el estudio, la escasez de información escrita sobre estos hospitales fue suplida por las entrevistas con los protagonistas. Resalta el contraste entre las políticas asimilacionistas del siglo XX y la situación actual de reposicionamiento social y político de los indígenas, que ha permitido algún avance en la apertura institucional hacia la interculturalidad en los servicios de salud, aunque todavía incipiente.

Los cambios en el hospital, de un periodo al otro, reflejan, además de la evolución de las teorías sobre la relación de los indígenas con la sociedad mayoritaria, la falta de continuidad y unidad de las políticas aplicadas. Por un lado, está el sector salud, orientado por el modelo biomédico excluyente, que históricamente no ha sido permeable a la participación de otras culturas médicas; por otro, la política indigenista que, en general, privilegia el abordaje integral e intersectorial y promueve la interculturaldiad y el conocimiento de la medicina tradicional.

Podemos constatar que a pesar del intento de un sector gubernamental representado principalmente por el INI, de poner en práctica ciertos aspectos de las políticas y acuerdos internacionales, como la intersectorialidad, el respeto y apoyo a la medicina tradicional y algunas adaptaciones interculturales en los servicios, no se avanzó lo suficiente en darle voz y autonomía a las comunidades para que puedan decidir su relación con la medicina biomédica. Esto puede explicarse por el poder del sector médico que sobrepasó al del INI, organismo que no tuvo durante su existencia mayor injerencia en los planes de estudio de los profesionales de la salud, ni en el modelo nacional de prestación de servicios. Su experiencia ha sido subutilizada por la SSA, no sólo para la transición del hospital de Cuetzalan, sino para el sistema de salud en general.

Observamos también que a pesar de los esfuerzos gubernamentales por el mejoramiento en la capacidad técnica para la prestación de servicios de salud y la prevención de la enfermedad en zonas indígenas como la de Cuetzalan, no se ha logrado superar el rezago epidemiológico de la población indígena, por las precarias condiciones de vida, a lo cual se suma la escasa perspectiva sociocultural en la planeación e implantación del modelo de atención.

Los servicios de salud siguen siendo, en términos generales, culturalmente homogéneos, impuestos desde arriba y excluyentes de otras alternativas diferentes al modelo médico hegemónico. Se sigue impartiendo educación en salud partiendo del supuesto de que los indios carecen de conocimientos sanitarios. La inclusión de la medicina indígena en algunas unidades de salud de la SSA (como los Hospitales Mixtos) y del IMSS-Oportunidades, representan, por lo tanto, un esfuerzo insuficiente en el logro de una verdadera medicina intercultural en el sistema de salud mexicano. ${ }^{23}$

Sin embargo, el gobierno federal actual abrió espacios institucionales para nuevas políticas de interculturalidad en los servicios de salud, las cuales se encuentran en etapa de diseño y ejecución incipiente, a cargo de la Dirección de Medicina Tradicional de la SSA. El interés del Poder Ejecutivo, favorece la abogacía por nuevos y mayores recursos, la apertura de espacios de participación y complementariedad, y la elaboración de propuestas sobre instancias e instrumentos jurídicos que respondan a las necesidades de salud intercultural. El haber constatado la influencia de los cambios en las políticas sociales nacionales e internacionales en las de- 
cisiones sobre salud sugiere que los decisores y los interesados deben estar alerta, ya que pueden ser, tanto oportunidades como amenazas para la construcción de modelos alternativos de prestación de servicios de salud a las comunidades indígenas, que contribuyan a la, denominada por Stavenhagen, ${ }^{26}$ liberación cultural autónoma, reclamada por las comunidades.

La composición intersectorial de la nueva Comisión para el Desarrollo de Pueblos Indígenas podría posibilitar una política unificada que impulse la construcción de modelos locales de salud participativos e interculturales, partiendo de las necesidades y particularidades étnicas y sociales, y garantizando el carácter decisorio, y no sólo consultivo, de la comunidad en la definición de su modelo.

En todo caso, Cuetzalan genera preguntas sobre el contexto, que deberían ser tomadas en cuenta en futuras investigaciones que pretendan aportar mayor información para la planeación de los servicios de salud en comunidades indígenas: ¿cómo lograr la incorporación real del enfoque intercultural en los programas nacionales de salud?, ¿cómo avanzar en un sistema nacional de salud incluyente de la diversidad terapéutica, que refleje la diversidad cultural?, ¿existen mejores alternativas de complementariedad entre medicina occidental y tradicional para estas zonas?, ¿cómo lograr que la experiencia del hospital de Cuetzalan sirva como fuente de decisiones en hospitales de otras regiones?, ¿qué viabilidad tendrá este hospital de mantenerse como "Mixto" una vez que termine el apoyo económico del Plan Puebla-Panamá?

Así como para Bonfil, ${ }^{27}$ la verdadera cultura mexicana sería la expresión libre de las diferentes culturas, la medicina mexicana en las zonas indígenas sería la libre expresión de la medicina biomédica y de la tradicional, dentro de un proceso de desarrollo integral autónomo. De esta manera, el sistema de salud de México respondería a las demandas de autonomía y respeto de los pueblos indígenas, apoyando el desarrollo de modelos de atención intercultural definidos por y no sólo para ellos. Esto redundaría en un mejor aprovechamiento de los recursos asignados y en una mayor satisfacción de los usuarios. El hospital de Cuetzalan y el proyecto de Hospitales Integrales con Medicina Tradicional constituyen un avance y un intento plausible por cumplir con algunos de los compromisos del Convenio 169 de la OIT y de las recomendaciones de la OPS/OMS, pero siguen siendo planeados, ejecutados y evaluados "desde arriba" por las instituciones gubernamentales, en procesos en los que las comunidades no tienen poder de decisión y apenas son consultadas.

A pesar del avance en la aceptación de los derechos indígenas y en su revaloración social en la última década, no se logra superar en la acción del Estado, y en una parte del gremio médico, la idea de que el respeto a las formas propias de prevención y tratamiento constituyen un rezago y no un enriquecimiento de la cultura médica nacional. Esto se refleja en que el sistema de salud, aunque acepta teóricamente que el efecto terapéutico de los medicamentos está ligado al contexto simbólico y emocional que tiene sentido en la cultura propia, ${ }^{9}$ sólo tolera la medicina tradicional en programas o proyectos específicos, ${ }^{28}$ y ha abierto algunos espacios de concertación, pero aún no se decide a incluir en el sistema de salud otras alternativas terapéuticas diferentes a la alopática.

Ganan espacio las ideas planteadas por Stavenhagen y otros, acerca de la pluriculturalidad como algo que enriquece y fortalece la cultura nacional, y de la obligación del Estado de fortalecer las culturas indígenas, como alternativa frente a las políticas homogeneizantes. ${ }^{15} \mathrm{~A}$ pesar de que la medicina indígena y la biomédica hayan sido calificadas como irreconciliables, existen ahora ejemplos nacionales e internacionales de logros en materia de servicios de salud interculturales con autonomía, como el de Makewe en Chile, ${ }^{*}$ el de Otavalo en Ecuador, y el de las comunidades autónomas de Chiapas, entre otros, que permiten vislumbrar la posibilidad no muy lejana de crear nuevos pactos que avancen en la creación de servicios de salud verdaderamente interculturales, que sirvan de ejemplo para el resto del país y del continente.

\section{Referencias}

1. Brachet-Márquez V. Reforma de la salud en A mérica Latina: aclarando el vínculo entre actores y estado. En: Solimano G, Isaacs E, ed. De la reforma para unos a la reforma para todos. Santiago de Chile: Editorial Sudamericana; 2000: 207-228.

2. Mooij J,VosV. Policy processes:An annotated bibliography on policy processes, with particular emphasis on India. Londres, UK: 0 verseas Development Institute.W orking paper 221. D isponible en: http:// www.odi.org.uk/publications/working_papers/wp221.pdf. [2003 julio 31]. 3. Rodríguez G, Gil J, García E. Metodología de la investigación cualitativa. Málaga: Editorial A lebrije;1990.

4. Gunderman H. El método de los estudios de caso. En: 0 bservar, escuchar y comprender. Sobre la tradición cualitativa en la investigación social.Torres ML, coord. México, DF: Editorial Porrúa/Flacso. 5. Duarte-Gómez MB. Medicina occidental y otras alternativas: ies posible su complementariedad? Reflexiones conceptuales. Cad Saude Publica 2003;19(2):109-118.

6. A guirre-Beltrán G. Antropología Médica. 0 bra Antropológica. Vol XIII. México, DF: Fondo de Cultura Económica;1994.

* Ibacache J. Modelo de atención de salud integral en el hospital Makawe. Santiago de Chile. Sf. Documento no publicado. 
7. Campos-N avarro R, Ruiz-Llanos A. A decuaciones interculturales en los hospitales para indios en la N ueva España. Gac Med Mex 2001; 37(6):595-608.

8. A guirre-Beltrán G. Programas de salud en la situación intercultural. 0 braAntropológica.Vol V. México, DF: Fondo de Cultura Económica;1994.

9. Bonfil G. México profundo. Una civilización negada. México, DF: Editorial Grijalbo; 1994.

10. Pozas R, Pozas I. Los indios en las clases sociales de México. 5a ed. México, DF: Siglo XXI; 1976.

11. Florez J. La revuelta por la democracia. Pueblos indios, política y poder en México. México, D F: Editorial El Atajo/Universidad Autónoma Metropolitana; 1998.

12. Brading D. El nacionalismo revolucionario, agotado. N ota periodística. La Jornada (México DF); 200314 de septiembre: p2. 13. Stavenhagen R. Problemas y perspectivas de los estados multiétnicos. Universidad de las $\mathrm{N}$ aciones Unidas. Serie anual de Conferencias \#3. Conferencia; 198627 de marzo; Japón.

14. Brachet-Márquez V. N acimiento, auge y decline del estado benefactor mexicano (1823-2000). Ponencia presentada en el seminario Social Policy in late industrializer:A comparative study of Latin America. United $N$ ations Research Institute for Social D evelopment; 2003 octubre 13-15. Santiago de Chile.

15. Gamio M. Forjando Patria. México, DF: Ed Porrúa;1960. 16. W arman A, N olasco M, Bonfil G, O livera M,Valencia E. De eso que llaman antropología mexicana. México, DF: Editorial N uestro Tiempo; 1970. 17.A guirre-Beltrán G, Pozas R. La política indigenista en México. Métodos y resultados. En: Medicina y salubridad. Instituciones indígenas en México actual.Tomo II. C ap V. 2ª edición. México, DF: IN I-SEP; 1973:151-186
18. Redfield R, Park M. D isease and its treatment in D zitas,Yucatán. W ashington, DC: Carnegie Institution; 1940.

19. Fajardo G, C arrillo A, N eri R. Perspectiva histórica de atención a la salud en México 1902-2002. México, DF: O PS/UN AM/ Sociedad Mexicana de Historia y Filosofía de la Medicina; 2002.

20. Hernández-Llamas H. H istoria de la participación del Estado en las instituciones de atención médica en México 1935-1980. En: 0 rtiz F, ed. Vida y muerte del mexicano. México, DF: Folios Ediciones; 1982:49-96. 21. 0 rganización Panamericana de la Salud/O rganización Mundial de la Salud. D eclaración de A Ima Ata. Alma A ta, U nión Soviética: O PS; 1978. 22. 0 rganización Internacional del Trabajo. La O IT y los pueblos indígenas y tribales. Folleto N úm. 8; 1994:46 pp. Disponible en: www.ilo.org. [2003 febrero 19].

23. Campos-N avarro R. La Medicina intercultural en hospitales rurales de A mérica Latina. Salud Problema 1999;Año 4(7):75-80.

24. Coordinación General del Plan Puebla Panamá. El Plan Puebla Panamá. Resumen Ejecutivo. 200328 de mayo. Disponible en: www.ciepac.org/ppp.htm. [2003 agosto 3].

25. Barreda A. Los peligros del Plan Puebla Panamá. En: Mesoamérica los ríos profundos: alternativas plebeyas al Plan Puebla Panamá. México, DF: Instituto Maya; 2001:133-201 Disponible en www.ciepac.org/ppp.htm. [2003 agosto 3].

26. Stavenhagen R. Problemas étnicos y campesinos. Ensayos. México, DF:IN I; 1980.

27. Bonfil G. Del indigenismo de la revolución a la antropología crítica. En: Eso que llaman antropología mexicana. México, D F:AEN AH; 1970: 39-65.

28. N igenda G, Mora-Flores G,Aldama-López S, O rozco-N uñez E. La práctica de la medicina tradicional en América Latina y El Caribe: el dilema entre regulación y tolerancia. Salud Publica Mex 2001;43:41-51. 